\title{
Protein Quantification by Selective Isolation and Fragmentation of Isotopic Pairs Using FT-ICR MS
}

\author{
Hannah Johnson, ${ }^{a}$ Stephen C. C. Wong, ${ }^{\text {a }}$ Deborah M. Simpson, ${ }^{b}$ \\ Robert J. Beynon, ${ }^{b}$ and Simon J. Gaskell ${ }^{\mathrm{a}}$ \\ ${ }^{a}$ Michael Barber Centre for Mass Spectrometry, School of Chemistry and Manchester Interdisciplinary \\ Biocentre, University of Manchester, Manchester, United Kingdom \\ ${ }^{b}$ Proteomics and Functional Genomics Research Group, Faculty of Veterinary Science, University of \\ Liverpool, Liverpool, United Kingdom
}

Isolation of tryptic peptide ions, along with their differentially labeled analogs derived from an artificial QconCAT protein, is performed using multiple correlated harmonic excitation fields in an FT-ICR cell. Simultaneous fragmentation of the isolated unlabeled and labeled peptide pairs using IRMPD yields specific $y$-series fragment ions useful for quantification. The mass increment attributed to stable isotope labeling at the C-terminus is maintained in the $\mathrm{C}$-terminal fragment ions, providing multiple measurements of labeled/unlabeled intensity ratios during highly selective detection. The utility of this approach has been demonstrated in the absolute quantification of components of an unfractionated chicken muscle protein mixture. (J Am Soc Mass Spectrom 2008, 19, 973-977) (C) 2008 American Society for Mass Spectrometry

$\mathrm{T}$ The absolute quantification of proteins at the global level is an important challenge that must be addressed, particularly in systems biology [1]. When modeling cellular pathways and networks it is important to quantify the constituent proteins. Relative quantification methods are designed to compare protein amounts in matched samples [2]. However, these methods are limited to identifying changes in the amount of a protein with respect to a second cellular state and samples vary even within control sets of the same organism [3].

When comparing samples analyzed at different times, using different instrumental platforms and within different laboratories, absolute quantification using a universal stable isotope-labeled internal standard of known concentration allows direct comparison. Currently, the most widely used methods rely on the well-established principles of stable isotope dilution techniques, using tryptic peptides as surrogate analytes for the proteins of interest [4]. Chemical synthesis and stable isotope incorporation of individual internal standards allow direct signal comparison, enabling the inference of protein quantities [4]. The production of a collection of internal standards in a single step has been enabled by the design of a DNA construct that is transcribed and translated into a protein concatamer, a technique referred to as QconCAT [5-7]. The produc-

Address reprint requests to Prof. Simon J. Gaskell, University of Manchester, School of Chemistry and Manchester Interdisciplinary Biocentre, John Garside Building, 131 Princess Street Manchester M1 7DN, UK. E-mail: simon.gaskell@manchester.ac.uk tion of a QconCAT protein thereby enables absolute quantification of multiple proteins using their peptide surrogates encoded within the QconCAT [7]. In a typical QconCAT workflow those proteins for which absolute quantification is required are defined and one or more specific reference peptides (Q-peptides) are chosen for each protein [5]. The DNA coding sequences for these peptides are concatenated, inserted into a vector, and expressed in Escherichia coli to form an artificial QconCAT protein. Growth of the transfected $E$. coli in stable isotope-containing media yields labeled QconCAT protein [5, 6]. To achieve absolute quantification, the QconCAT protein is introduced, in a known amount, into the biological sample of interest and proteins are digested with trypsin to yield Qpeptides alongside native peptides. The light-to-heavy ratio $(\mathrm{L} / \mathrm{H})$ of native peptide to the internal reference peptide is calculated following mass spectrometric analysis, allowing inference of the absolute concentration of each native peptide and thus the protein from which it was derived.

The use of tandem mass spectrometry (MS/MS) in relative quantification has previously been demonstrated to increase both the specificity and sensitivity of analyses $[8,9]$. In this study, we use multiple correlated harmonic excitation fields (multi-CHEFs) as a method of ion isolation within a Fourier transform ion cyclotron resonance (FT-ICR) MS cell. Multi-CHEFs are tailored waveforms that are stitched together within the acquisition software to allow selective isolation of multiple precursor ions according to their $m / z[10,11]$. To achieve 
quantification, isotopic variants of a peptide of interest (both naturally occurring and stable isotope-containing) are co-isolated from the unfractionated ion population. Nonselective activation of these isolated ions, using infrared multiphoton dissociation (IRMPD) [12], generates quantitative and qualitative information pertaining to the differentially labeled Q-peptide pairs. Stable isotope incorporation using ${ }^{15} \mathrm{~N}$ or $\left[{ }^{13} \mathrm{C}_{6}\right]-\mathrm{K} / \mathrm{R}$ introduces a mass increment of 12-24 and $6 \mathrm{Da}$, respectively, for each Q-peptide. Where $\left[{ }^{13} \mathrm{C}_{6}\right]-\mathrm{K} / \mathrm{R}$ has been used, specific quantitative measurements can be derived from the $y$-series fragment ions, which differ by a constant 6 Da. Labeling with ${ }^{15} \mathrm{~N}$ produces a varying mass difference between labeled and unlabeled counterparts in both $b$ - and $y$-series ions, making data processing more challenging.

This approach significantly reduces the confounding influence of any peptides whose precursor ion signal overlaps with the analytes of interest. Because of the complexity of biological samples, it is important to validate the quantification calculated using the precursor ion population alone if using limited or no peptide/ protein separation pre-MS. In our work, two independently selected isotopic variants (arising from labeled QconCAT and native chicken protein) constitute a precursor ion population that is subsequently fragmented by IRMPD [12]. We have demonstrated the utility of this approach for absolute quantification of proteins within unfractionated chicken muscle protein.

\section{Experimental}

\section{Sample Preparation}

Chicken skeletal muscle protein and stable isotope $\left[{ }^{15} \mathrm{~N}\right]-$ and $\left[{ }^{13} \mathrm{C}_{6}\right]-\mathrm{K} / \mathrm{R}$-labeled chicken muscle QconCAT protein were prepared and quantified as described previously $[6,7]$. Labeled QconCAT was added in known amounts to unfractionated chicken muscle protein sample for quantitative analysis. Proteins were reduced, alkylated (with DL-dithiothreitol and iodoacetamide, respectively), and incubated with trypsin at $37^{\circ} \mathrm{C}$ for $18 \mathrm{~h}$. Peptides were desalted postdigestion using a C18 peptide trap (Presearch Ltd, Hampshire, UK) and stored at $-20{ }^{\circ} \mathrm{C}$ until MS analysis.

\section{FT-ICR Mass Spectrometry}

Experiments were performed on a 9.4-T FT-ICR mass spectrometer (Apex III'TM; Bruker Daltonics, Billerica, MA, USA) with an Apollo electrospray source and Synrad 48 series IR laser ( $\lambda=1060 \mu \mathrm{m}$; Synrad, Mukilteo, WA, USA). Selective isolation of ions was achieved using multi-CHEF [11], where each "notch" was centered on the principal isotopic variant of the selected precursor ion. Pulsed argon gas was used to facilitate the confinement of ions by collisional cooling, thereby maximizing overlap of the ion cloud with the IR laser beam. Multi-CHEF isolation of isotopic envelopes cor- responding to unlabeled and labeled peptide variants was achieved with $80 \%$ efficiency. Losses increased to $50 \%$ when pulsed gas was not used. The co-isolated pairs of native and stable isotope-bearing Q-peptide ions were excited in a nonselective manner to promote fragmentation. The calculation of light/heavy isotope ratios was performed manually using the peak height of the principal isotopic peaks of the light and heavy analogues.

\section{Results and Discussion}

Unlabeled and $\left[{ }^{13} \mathrm{C}_{6}\right]-\mathrm{K} / \mathrm{R}$ QconCAT protein were mixed in a 1:1 ratio, digested with trypsin, and analyzed by FT-ICR MS. Each pair of tryptic peptide $[\mathrm{M}+2 \mathrm{H}]^{2+}$ ions displayed a $6 \mathrm{Da}$ (3 Th) difference, consistent with the presence of one lysine or arginine residue. Various isolation notch widths were evaluated with respect to the reproducibility of the subsequent determination of the signal ratio of labeled to unlabeled analogues. Achievement of the narrowest precursor ion isolation window (and therefore highest effective resolution of the precursor ion) was achieved using the highest excitation energy. Initial experiments involved the use of a double-notch waveform to effect specific isolation of the principal isotopic variants of the unlabeled and labeled peptide ions. This, however, resulted in significant loss of signal intensity, which was proportionately greater for the lower mass (higher frequency) ion population, thereby confounding quantitative analysis. Thus multi-CHEF was used to isolate the isotopic envelope of both the natural and stable isotope-incorporating peptide analogues.

The combined precursor ion population was then subjected to IRMPD, producing a series of singly charged $y$-ion pairs, with a separation of 6 Th (attributed to the retention of the C-terminal label), displaying $\mathrm{L} / \mathrm{H}$ ratios useful for quantification. For each pair of peptide analogs, a preisolation $\mathrm{L} / \mathrm{H}$ ratio was calculated along with the $\mathrm{L} / \mathrm{H}$ ratio upon isolation and the mean $\mathrm{L} / \mathrm{H}$ ratio of the $y$-series fragment ion doublets. The $\mathrm{L} / \mathrm{H}$ ratios for 10 peptide pairs pre- and postisolation were $0.97 \pm 0.10$ and $0.96 \pm 0.10(n=36)$ (where $n$ is the number of analyses), indicating that the isolation process as applied had no effect on quantification. Postfragmentation, the mean $\mathrm{L} / \mathrm{H}$ ratio calculated for the fragment ions in this dataset was $1.08 \pm 0.11$. For each product ion spectrum the mean of the coefficient of variation for the $y$-series fragment ions used was $14 \pm$ $7 \%$, where four to eight fragment ion pairs were used for quantification within each experiment. For a single peptide ion pair of $m / z$ 929.96/932.97 (T21), the L/H ratios for a 1:1 mixture of light and heavy QconCAT were $0.98 \pm 0.10$ and $0.95 \pm 0.04(n=14)$, pre- and postisolation, respectively. For this peptide analyzed once, the resulting fragment ions produced the $\mathrm{L} / \mathrm{H}$ ratio $1.05 \pm 0.07(f=7)$ (where $f$ is the number of $y$-series fragment ion doublets). For the same peptide 
pair analyzed 14 times, the mean of the mean fragment ion ratios was $1.07 \pm 0.14(n=14)$.

To assess this approach for determination of isotope ratios across a broader range, $\left[{ }^{13} \mathrm{C}_{6}\right]-\mathrm{K} / \mathrm{R}$-incorporating QconCAT was mixed in a ratio of 1:10 with unlabeled QconCAT, digested with trypsin, and analyzed by FT-ICR MS. The L/H ratios obtained preisolation, upon isolation, and postfragmentation, for three peptides, were $0.105 \pm 0.008,0.104 \pm 0.003$, and $0.130 \pm 0.006$ $(n=7)$. A mean $\mathrm{CV}$ of $22 \pm 2.4 \%$ was calculated for each individual product ion spectrum, where three to four $y$-series fragment ion pairs were used for quantification. The observation of increased $\mathrm{L} / \mathrm{H}$ ratios and higher $\mathrm{CV}$ values reflected reduced signal to noise of the unlabeled counterpart. These data suggest satisfac- tory accuracy for the determination of ratios 1:10, although the precision is reduced.

This approach was further validated using unlabeled and $\left[{ }^{15} \mathrm{~N}\right]$-labeled QconCAT mixed at ratios of 7:1 before digestion with trypsin. Because of the nature of this labeling method the resulting $\left[{ }^{15} \mathrm{~N}\right]$-labeled peptides have mass differences in comparison with their unlabeled counterparts, which are dependent on amino acid composition. For five pairs of labeled and unlabeled peptides the pre- and postisolation and postfragmentation ratios were $6.47 \pm 2.39,5.98 \pm 2.15$, and $6.25 \pm$ $0.03(n=5)$. For T19, the labeled peptide isotope pattern overlaps with that of unlabeled T20 peptide; this prevents accurate determination of the $\mathrm{L} / \mathrm{H}$ ratios from conventional mass spectra (not MS/MS). The apparent

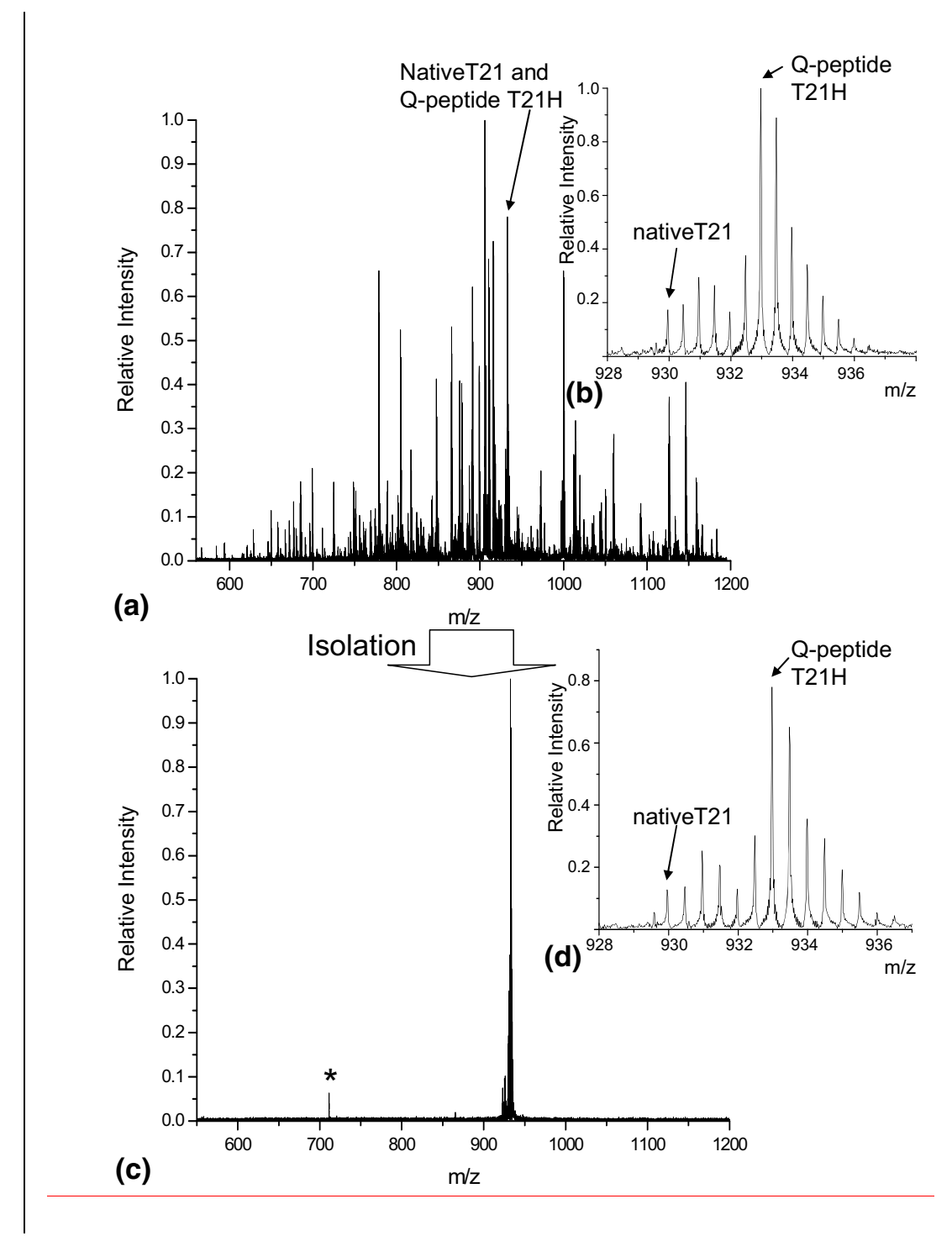

Figure 1. FT-ICR analyses of a tryptic digest of unfractionated chicken muscle protein supplemented with $\left[{ }^{13} \mathrm{C}_{6}\right]-\mathrm{K} / \mathrm{R}$ QconCAT protein. (a) Mass spectrum of unfractioned chicken muscle protein digested with trypsin. (b) Expanded region corresponding to doubly protonated FGVEQNVPMVFASFIR and its labeled Q-peptide counterpart. (c) Mass spectrum of digested, unfractioned, chicken muscle protein after selective isolation of ions within the range, $\mathrm{m} / \mathrm{z}$ 930-933. The peak labeled * at approximately $\mathrm{m} / \mathrm{z} 710$ represents electronic noise. (d) Expanded region, $m / z$ 930-933, after isolation. 
$\mathrm{L} / \mathrm{H}$ ratio obtained pre- and postisolation for T19 was 1.22 and 1.19 , respectively. Following fragmentation the composite product ion spectrum included a $y$-ion series specific to T19. Two members of this series $\left(y_{2}\right.$ and $\left.y_{3}\right)$, showing no overlap with other fragments, were selected for quantification yielding $\mathrm{L} / \mathrm{H}$ ratio of 6.21 and 7.57 (average 6.89). Thus, in this instance the determination of isotope ratios was confounded when using conventional MS analysis alone (without chromatographic separation). Deconvolution of overlapping signals was achieved by precursor ion isolation and fragmentation, enabling accurate quantification. Furthermore, the precision of isotope ratio determination was significantly improved when using fragment ion data.

The same MS/MS approach was utilized for the quantification of an unfractionated chicken cell lysate, with $\left[{ }^{13} \mathrm{C}_{6}\right]-\mathrm{K} / \mathrm{R}$-labeled QconCAT as the internal standard. Chicken muscle QconCAT (93 nmol/g of chicken muscle) was added to chicken muscle soluble protein from a 30-day-old broiler chicken. The mixture was digested with trypsin and analyzed by FT-ICR MS. The conventional mass spectrum (Figure 1a) indicates the complexity of the unfractionated cell lysate, with approximately 500 peaks being readily observed. Figure $1 \mathrm{~b}$ shows an expansion of that portion of the spectrum expected to include both labeled and unlabeled analogues of the protonated T21 peptide (sequence FGVEQNVPMVFASFIR, designed for the quantification of chicken pyruvate kinase). The complexity of the apparent isotope patterns suggests the overlapping detection of multiple species, implying the need for detection of enhanced selectivity. The determination of signal intensity ratio $(\mathrm{L} / \mathrm{H})$ for the $\mathrm{T} 21$ peptide using conventional MS (Figure 1b) gave an apparent value of 0.17 , but this is expected to be inaccurate in view of the overlapping signals observed. The entire isotopic envelopes of native chicken peptide T21 and its $\left[{ }^{13} \mathrm{C}_{6}\right]$ $\mathrm{R}$-analogue were then isolated (Figure 1c). The relative signal intensities corresponding to the isolated species (Figure 1d) reproduced those observed in the original spectrum (Figure 1b). Upon fragmentation of the isolated species, a series of six $y$-fragment ion pairs were observed, each separated by 6 Th (Figure 2). A labeled/ unlabeled signal ratio was determined for all pairs except that corresponding to $y_{1}$; this was excluded because of a possible contribution from other tryptic peptides not separated in the mass spectrometric analysis. A mean ratio of 0.24 was calculated for the remaining five $y$-series ions (Figure 2 ) with a CV of $10 \%$; this ratio is significantly different from that determined from the conventional mass spectrum, confirming the benefit of enhanced selectivity of analysis. It is noteworthy that the apparent $\mathrm{L} / \mathrm{H}$ ratio for $y_{1}$ was 0.69 , suggesting its origin from multiple peptide precursors. This particular experiment was repeated and $\mathrm{L} / \mathrm{H}$ ratios preisolation, upon isolation, and the $\mathrm{L} / \mathrm{H}$ ratio of the fragment ions were calculated at $0.16 \pm 0.01,0.16 \pm 0.01$, and $0.26 \pm 0.04$ respectively $(n=6)$. These data indicate an

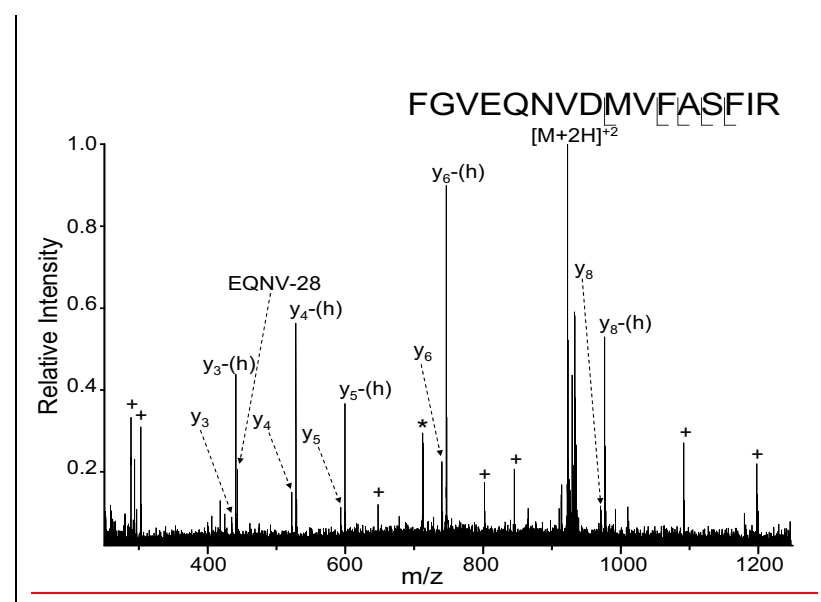

Figure 2. Product ion spectrum showing ions derived from doubly protonated tryptic peptide, T21 (FGVEQNVPMVFASFIR) and its labeled Q-peptide partner following IRMPD. $y$-ions derived from T21 are noted; " $\mathrm{y}_{n}(\mathrm{~h})$ " indicates a ${ }^{13} \mathrm{C}_{6}$-containing fragment. The peaks labeled "+" are not derived from FGVEQNVPMVFASFIR and are attributed to other precursor ions of similar $\mathrm{m} / \mathrm{z}$. The peak labeled * at approximately $\mathrm{m} / \mathrm{z} 710$ represents electronic noise.

absolute quantity of pyruvate kinase of $24.2 \pm 4.1 \mathrm{nmol} / \mathrm{g}$ of chicken muscle.

\section{Conclusions}

FT-ICR MS using the multi-CHEF technique has been introduced to simultaneously isolate and fragment light and heavy Q-peptide pairs. Furthermore, this approach has been used to quantify unfractionated native chicken muscle peptides alongside $\left[{ }^{13} \mathrm{C}_{6}\right]-\mathrm{K} / \mathrm{R}$-labeled internal standards to achieve an increase in specificity. Isolation of ions of interest using multi-CHEF decreases ion interference, allowing the production of specific $y$-series fragment ions by IRMPD. Quantification using these $y$-series ions allows increased accuracy of quantification when there are overlapping species present at the same $\mathrm{m} / \mathrm{z}$. The isolation of multiple ions within an ICR cell can also be achieved using stored waveform inverse Fourier transform (SWIFT) [13]. The implementation of SWIFT as an alternative approach to ion isolation for absolute quantification is in progress.

In summary, we have reported an approach to the absolute quantification of proteins using MS/MS, which exploits the selective ion isolation capabilities of FT-ICR. This approach is of particular value when the complexity of the mixture to be analyzed is such that accurate and precise quantification is compromised when using conventional MS detection. We anticipate selective use of this method, for validation of data produced using less rigorous approaches.

\section{Acknowledgments}

This work was supported by the UK Engineering and Physical Sciences Research Council (reference EP/D013615/1) and the 
Biotechnology and Biological Sciences Research Council (reference BB/D013615/1). The authors thank the Roslin Institute, Edinburgh, UK for rearing birds and collecting muscle samples, Jennifer Rivers (University of Liverpool) for the preparation of the chicken muscle protein lysate, and Drs. Claire Eyers and Sarah Hart for useful discussions.

\section{References}

1. Aebersold, R.; Mann, M. Mass Spectrometry-Based Proteomics. Nature 2003, 422, 198-207.

2. Ross, P. L.; Huang, Y. N.; Marchese, J. N.; Williamson, B.; Parker, K.; Hattan, S.; Khainovski, N.; Pillai, S.; Dey, S.; Daniels, S.; Purkayastha, S.; Juhasz, P.; Martin, S.; Bartlet-Jones, M.; He, F.; Jacobson, A.; Pappin, D. J. Multiplexed Protein Quantitation in Saccharomyces cerevisiae Using Amine-Reactive Isobaric Tagging Reagents. Mol. Cell. Proteomics 2004, 3, 1154-1169.

3. Gan, C. S.; Chong, P. K.; Pham, T. K.; Wright, P. C. Technical, Experimental, and Biological Variations in Isobaric Tags for Relative and Absolute Quantitation (iTRAQ). J. Proteome Res. 2007, 6, 821-827.

4. Gerber, S. A.; Rush, J.; Stemman, O.; Kirschner, M. W.; Gygi, S. P. Absolute Quantification of Proteins and Phosphoproteins from Cell Lysates by Tandem MS. Proc. Natl. Acad. Sci. U.S.A. 2003, 100, 69406945.

5. Beynon, R. J.; Doherty, M. K.; Pratt, J. M.; Gaskell, S. J. Multiplexed Absolute Quantification in Proteomics Using Artificial QCAT Proteins of Concatenated Signature Peptides. Nat. Methods 2005, 2, 587-589.
6. Pratt, J. M.; Simpson, D. M.; Doherty, M. K.; Rivers, J.; Gaskell, S. J.; Beynon, R. J. Multiplexed Absolute Quantification for Proteomics Using Concatenated Signature Peptides Encoded by QconCAT Genes. Nat. Protocols 2006, 1, 1029-1043.

7. Rivers, J.; Simpson, D. M.; Robertson, D. H. L.; Gaskell, S. J.; Beynon, R. J. Absolute Multiplexed Quantitative Analysis of Protein Expression during Muscle Development Using QconCAT. Mol. Cell. Proteomics 2007, 6, 1416-1427.

8. Warwood, S. Mohammed, S. Cristea, I. M. Evans, C: Whetton, A. D. Gaskell, S. J. Guanidination Chemistry for Qualitative and Quantitative Proteomics. Rapid Commun. Mass Spectrom. 2006, 20, 3245-3256.

9. Panchaud, A.; Guillaume, E.; Affolter, M.; Robert, F.; Moreillon, P. Kussmann, M. Combining Protein Identification and Quantification C-Terminal Isotope-Coded Tagging Using Sulfanilic Acid. Rapid Commun. Mass Spectrom. 2006, 20, 1585-1594.

10. de Koning, L. J.; Nibbering, N. M. M.; van Orden, S. L.; Laukien, F. H. Mass Selection of Ions in a Fourier Transform Ion Cyclotron Resonance Trap Using Correlated Harmonic Excitation Fields (CHEF). Int. J. Mass Spectrom. Ion Process. 1997, 165-166, 209-219.

11. Kruppa, G.; Schnier, P. D.; Tabei, K.; van Orden, S.; Siegel, M. M. Multiple Ion Isolation Applications in FT-ICR MS: Exact-Mass MS Internal Calibration and Purification/Interrogation of Protein-Drug Complexes. Anal. Chem. 2002, 74, 3877-3886.

12. Little, D. P.; Speir, J. P.; Senko, M. W.: O'Connor, P. B.; McLafferty, F. W. Infrared Multiphoton Dissociation of Large Multiply Charged Ions for Biomolecule Sequencing. Anal. Chem. 1994, 66, 2809-2815.

13. Chen, L.; Wang, T. C. L.; Ricca, T. L.; Marshall, A. G. Phase-Modulated Stored Waveform Inverse Fourier Transform Excitation for Trapped Ion Mass Spectrometry. Anal. Chem. 1987, 59, 449-454. 